\title{
Original
}

\section{Immunolocalization of Axin2 and p-Smad3 in Developing Rat Molar Germ}

\author{
Mitsuko Moriguchi' ${ }^{1)}$, Marie Yamada', Yasuo Miake' ${ }^{1)}$ and Mayu Koshika ${ }^{1)}$ \\ 1) Department of Ultrastructural Science, Tokyo Dental College, Chiba, Japan \\ 2) Department of Physical Therapy, School of Health Science, Niigata University of Health and Welfare, Niigata, Japan
}

(Accepted for publication, February15, 2012)

\begin{abstract}
Wnt is involved in odontoblast and ameloblast differentiation and matrix protein expression in dentin and enamel, but there have been only a few reports on Axin2 in the Wnt signaling pathway. Axin2 promotes Smad3 activation in the transforming growth factor beta (TGF-ß) signaling pathway, and the presence of crosstalk between the Wnt and TGF-ß signaling pathways has been reported, but there have been no reports on that in odontogenic cells. Thus, we examined immunohistochemically the factors in the 2 signaling pathways using serial sections of rat first molar germ at embryonic day 19 and 10 days after birth to investigate whether there is cross-talk between the 2 signaling pathways through Axin2 and Smad3. Factors in the Wnt signaling pathway: Wnt10, Dishevelled (Dvl), and Axin2, and those in the TGF-ß signaling pathway: TGF-ß receptor 1 (TGF-ßR1), Smad3, and its active type, phosphorylated Smad3 (p-Smad3), showed similar immunoreactions; in detail, odontoblasts possessing secretory function, rather than dental papilla cells and preodontoblasts, and secretorystage ameloblasts, rather than inner enamel epithelium, showed stronger reactions, whereas the reaction of maturation-stage ameloblasts was very weak. In addition, the reaction of Axin2 was completely consistent with that of pSmad3, and odontoblasts and secretory ameloblasts showed the strongest positive reactions. Accordingly, it was suggested that the TGF- $ß$ signaling pathway as well as Dvl- and Axin2-mediated Wnt10 signaling pathway may are involved in odontoblast and ameloblast differentiation and the secretory function of these cells. In addition, the reaction of Axin2 was completely consistent with that of pSmad3, and odontoblasts and secretory ameloblasts showed the strongest positive reactions, suggesting that a cross-talk between the 2 signaling pathways through Axin2 and Smad3 may be involved in the odontoblast and ameloblast differentiation and secretory function of these cells.
\end{abstract}

Keywords: Immunolocalization, Developing rat molar germ, Axin2, p-Smad3

\section{Introduction}

In the Wnt/ $\beta$-catenin pathway, when Wnt binds to its receptors, Frizzled and low-density lipoprotein receptor-related protein 5/6 (LRP5/6), glycogen synthase kinase-3 beta (GSK-3ß) activity is inhibited through Dishevelled (Dvl), and scaffold protein of $\beta$ catenin destruction complex, Axin, becomes unstable due to the inactive-type GSK-3 $\beta$. The complex is then destroyed and releases $\beta$-catenin, resulting in its intracellular accumulation. Accumulated $\beta$-catenin is considered to transfer to the nucleus, bind to transcript factor; lymphoid enhancer-binding factor / T cell factor (LEF/ $\mathrm{TCF}$ ), and induce and regulate gene expression ${ }^{1)}$. Axin2 is a negative regulator of the Wnt signaling pathway ${ }^{2}$. Under Wnt signals, it is released from the complex and binds to LRP5/6, leading to Axin degradation ${ }^{3}$. In contrast, the $\mathrm{Wnt} / \beta$-catenin pathway is known to induce Axin2 mRNA transcription ${ }^{4}$, and

Correspondence to: Mitsuko Moriguchi, PhD. Department of Ultrastructural Science, Tokyo Dental College, 1-2-2 Masago Mihama-ku, Chiba 261-8502, Japan. Tel: +81-43-270-3755, Fax: +81-43-270-3756, Email: moriguti@tdc.ac.jp
Axin2 is considered to control Wnt signaling by forming a negative feedback loop ${ }^{5}$. Regarding the involvement of Wnt in tooth development, many studies have reported its involvement in the early stage of tooth germ formation, and it is considered to initiate tooth germ formation and control proliferation and differentiation of tooth germ epithelial cells and mensenchymal cells ${ }^{6), 7), 8), 9)}$. On the other hand, in the late bell stage in which dentin and enamel are formed, Wnt-10a mRNA is expressed in preodontoblasts and odontoblasts and dentin sialophosphoprotein (Dspp) mRNA is expressed by odontoblasts, on the basis of which Wnt-10a has been reported to be involved in odontoblast differentiation and dentin formation by regulating $\mathrm{Dspp}^{10}$. It has been reported that Wnt-10a and Axin2 appeared in the incisor tooth germ ameloblasts $^{11)}$, Wnt10, Dvl, inactive-type GSK-3 $\beta$ and $\beta$-catenin distributed in secretory-stage ameloblast and progenitors of the more tooth germ ${ }^{12)}$, and $\beta$-catenin/LEF1 activates the appearance of enamelin in ameloblast-like cells ${ }^{13}$, indicating involvement of the Wnt signaling pathway in the ameloblast differentiation and 

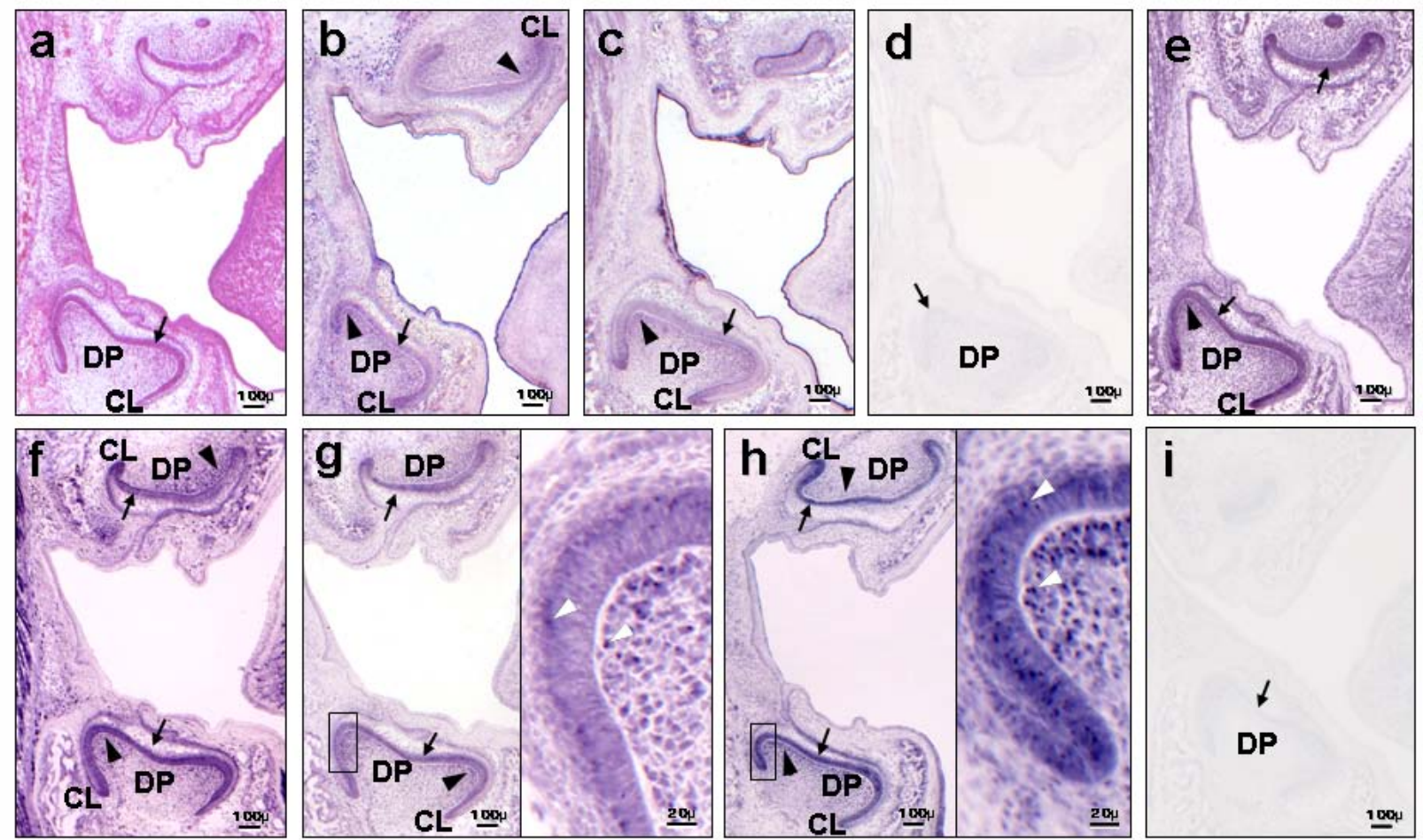

Figure 1. Immunohistochemical observation of the rat first molar germ on embryonic day 19.

a Hematoxylin and eosin (H-E) staining. Inner enamel epithelium (arrows) and mesenchymal cells of the dental papilla distributed densely along this epithelium are observed. b, c Staining with anti-Wnt10 (b) or anti-Dvl (c) was positive in the inner enamel epithelium and in the cervical loop. In the dental papilla, positive reactions were observed in mesenchymal cells in contact with the enamel epithelium. $d$ Negative control for figures (b) and (c). No clear reaction is noted. e, f Staining with anti-Axin2 (e) or anti-TGF-ß-R1 (f) was strongly positive in the inner enamel epithelium and in the cervical loop. Moderate reactions were observed in the dental papilla cells in contact with the enamel epithelium. g, h Staining with anti-p-Smad3 (g) or anti-Smad3 (h) was also strongly positive in the inner enamel epithelium and in the cervical loop. In the dental papilla, moderate reactions were also observed in mesenchymal cells in contact with the enamel epithelium. These stainings were strongest in the nucleus (white arrowhead). The boxed areas are enlarged to the right base of each panel. i Negative control for figures (e) - (h). No signal was detected. Arrows Inner enamel epithelium. Black arrowheads Dental papilla cells in contact with the enamel epithelium. DP Dental papilla,

CL Cervical loop. Bars, 100 ìm or $201 ̀ m$.

matrix protein secretion in enamel. Regarding the TGF- $\beta$ signaling pathway, the Smad pathway is well known, in which Smad3 is activated through TGF- $\beta$-R1, and active-type Smad3 and Smad4 form a complex, move into the nucleus, and regulate gene expression, through which the action is exhibited. Regarding tooth development, it has been reported that TGF- $\beta$ and its genes and receptor are localized in the inner enamel epithelium, cervical loop, ameloblasts, and odontoblasts ${ }^{14), 15), 16), 17)}$, and TGF- $\beta 1$ induces ameloblast and odontoblast differentiation ${ }^{18), 19)}$, which is involved in the production of matrix proteins, such as amelogenin, enamelysin, collagen, and Dspp 20), 21), 22), 23), 24). It has also been reported that Smad3 and Smad4 are distributed in inner enamel epithelium and dental mesenchyme and regulate tooth development ${ }^{24), 25)}$, and that Smad3 is necessary for mineralization of enamel ${ }^{26)}$.

Reportedly, there is cross-talk between the Wnt and TGF- $\beta$ signaling pathways. It has been reported that interaction between Axin and Smad3 promotes Smad3 activation in the TGF- $\beta$ signaling pathway ${ }^{27)}$, and that Axin and active-type GSK3- $\beta$ degrade Smad3 by phosphorylation and modulate TGF-ß signaling by controlling Smad3 stability ${ }^{28}$. However, there have been no reports on investigations of cross-talk between the 2 signaling pathways in tooth development. In this study, to investigate whether cross-talk between the Wnt and TGF- $\beta$ signaling pathways is involved in ameloblast and odontoblast differentiation and the function of these cells, we immunohistochemically examined the distributions of factors involved in the Wnt signaling pathway (Wnt10, Dvl, and Axin2) and in the TGF- $\beta$ signaling pathway (TGF- $\beta$-R1, Smad3, and p-Smad3) in the early bell stage immediately before differentiation of these cells, as well as in the late bell stage in which hard tissue formation is underway, using rat molar tooth germ.

\section{Materials and Methods}

\section{Tissue preparation}

The experiment was performed using Sprague-Dawley (SD) rats at embryonic day 19 and 10 days after birth, in accordance with the Guidelines for the Handling of Experimental Animals of 
Mitsuko Moriguchi et al.: Axin2 and p-Smad3 in Molar Germ
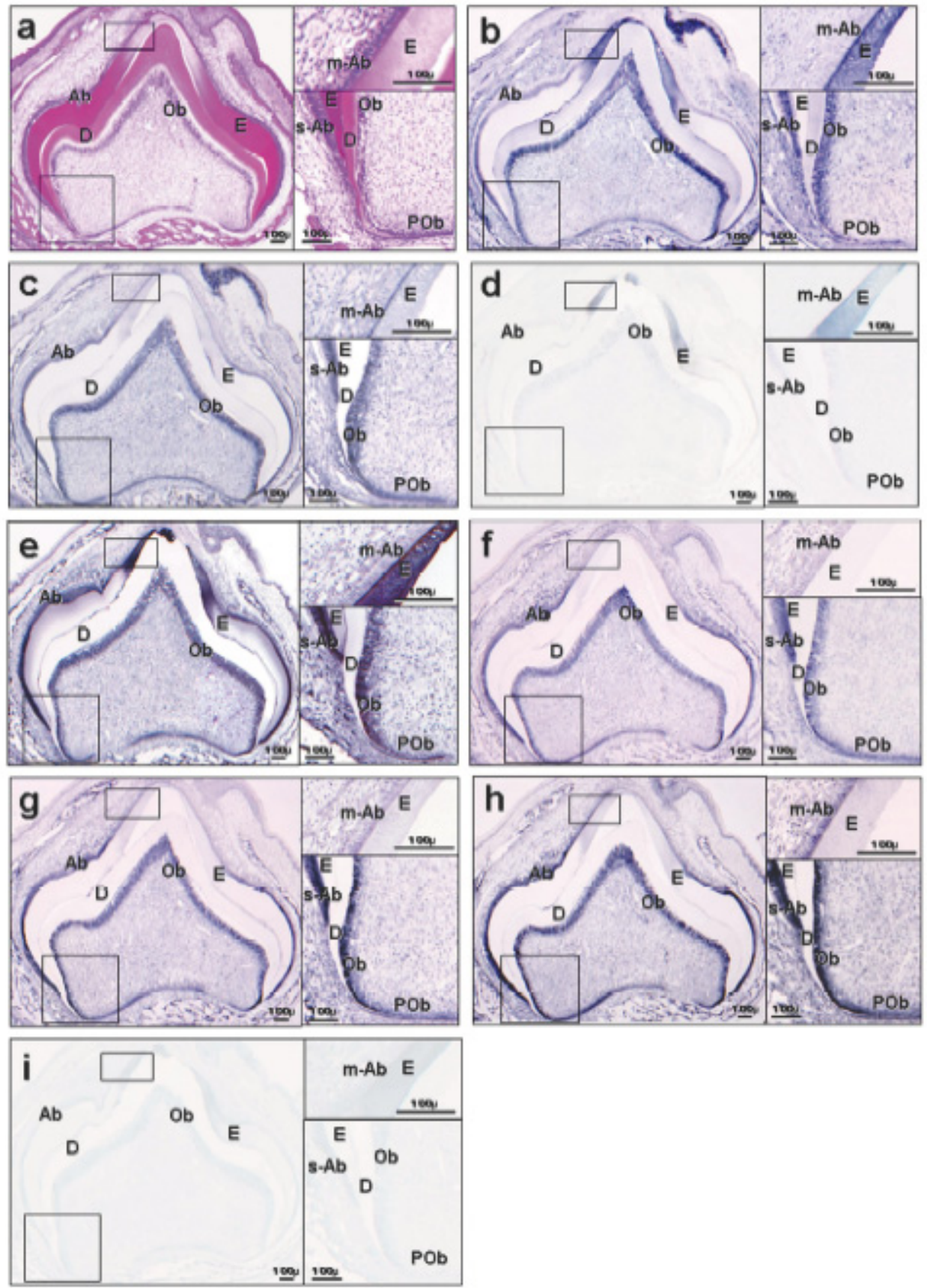

Figure 2. Immunohistochemical observation of the rat maxillary first molar germ 10 days after birth.

a H-E staining. Enamel, dentin, and Hertwig's epithelial sheath had formed. Secretory ameloblasts and odontoblasts were distributed, and differentiation from secretory ameloblasts into maturation ameloblasts and from preodontoblasts into odontoblasts was observed near the cusp or apical end. b, c Staining with anti-Wnt10 (b) or anti-Dvl (c) was strongly positive in secretory ameloblasts and odontoblasts, and also moderate in preodontoblasts, but weak in maturation ameloblasts, showing stronger reactions with differentiation into odontoblasts, and weaker reactions from secretory ameloblasts to maturation ameloblasts. d Negative control for figures (b) and (c). No clear reaction is noted. e Staining with anti-Axin2 was most strongly positive in the secretory ameloblasts and odontoblasts, and also moderate in preodontoblasts, showing stronger reactions with differentiation into odontoblasts and weaker reactions from secretory ameloblasts to maturation ameloblasts. f Staining with anti-TGF- $\beta$-R1 was strongly positive in the secretory ameloblasts and odontoblasts, but showed decreased reaction in maturation ameloblasts or preodontoblasts. g, $\mathrm{h}$ Staining with anti-p-Smad3 (g) or anti-Smad3 (h) was most strongly positive in the secretory ameloblasts and odontoblasts, and also moderate in preodontoblasts, showing stronger reactions with differentiation into odontoblasts and weaker reactions from secretory ameloblasts to maturation ameloblasts. These findings almost agreed with those for Axin2. i Negative control for figures (e) - (h). No signal was detected. Ab ameloblast, E enamel, D dentin, Ob odontoblast, m-Ab maturation ameloblast, s-Ab secretory ameloblast, POb preodontoblast. Bars 100ìm. The boxed areas are enlarged to the right of each panel. 
J.Hard Tissue Biology Vol. 21(2):113-120, 2012

Table 1. Immunohistochemical distribution of Wnt10, Dvl, Axin2, TGF-ß-R1, p-Smad3, and Smad3 during rat tooth development.

\begin{tabular}{|c|c|c|c|c|c|c|}
\hline Cell layer & Wnt10 & Dvl & Axin2 & TGF-ß-R1 & p-Smad3 & Smad3 \\
\hline Inner enamel epithelium & + & + & ++ & ++ & ++ & ++ \\
\hline $\begin{array}{l}\text { Dental papilla cells } \\
\text { (contact with the ename epithelium) }\end{array}$ & + & + & + & + & + & + \\
\hline Cervical loop & + & + & ++ & ++ & ++ & ++ \\
\hline Ameloblasts (secretory stage) & ++ & ++ & +++ & +++ & +++ & +++ \\
\hline Ameloblasts (maturation stage) & \pm & \pm & \pm & \pm & \pm & + \\
\hline Preodontoblasts & + & + & + & + & + & + \\
\hline Odontoblasts & ++ & ++ & +++ & ++ & +++ & +++ \\
\hline
\end{tabular}

+++, strongest immunoreactivity; ++, strong immunoreactivity; +, moderate immunoreactivity; $\quad \pm$, weak immunoreactivity.

Tokyo Dental College. The rats were fixed by perfusion or immersion in $0.01 \mathrm{M}$ periodic acid-0.075 M lysine-2\% paraformaldehyde (PLP). Samples were fixed and decalcified with triethylammonium ethylenediamine tetraacetic acid (EDTA) in $80 \%$ ethanol ${ }^{29}$. The maxillary first molar tooth germ with surrounding tissues was removed, embedded in paraffin, and a series of longitudinal sections of 5 ìm thickness were prepared. Some of the sections were treated with hematoxylin and eosin (H-E) staining.

\section{Immunostaining}

The paraffin sections were deparaffinized, and the antigen was activated and unmasked by irradiating the sections twice with 400-W microwaves in 0.01\% citric acid buffer (PH 6.0) for 15 min. Free aldehyde radicals were removed by immersing the sections in $0.1 \%$ glycine phosphate-buffered saline (PBS) for 30 $\mathrm{min}$, and the endogenous peroxidase activity was eliminated by immersing them in $0.3 \% \mathrm{H}_{2} \mathrm{O}_{2}, 0.1 \% \mathrm{NaN}_{3}$, and $\mathrm{PBS}$ for $30 \mathrm{~min}$. Blocking was performed for $1 \mathrm{~h}$ with normal goat or rabbit serum (1:10). The sections were incubated overnight at room temperature with the following primary antibodies diluted in $1 \%$ bovine serum albumin (BSA) PBS: Wnt10 (C-19) (anti-Wnt10b, C-terminus, goat polyclonal antibody; Santa Cruz Biotechnology, Santa Cruz, CA; 1:50) (detectable Wnt10a and Wnt10b), Dvl (G-19) (antiDvl, N-terminus, goat polyclonal antibody; Santa Cruz Biotechnology, Santa Cruz, CA; 1:50), Axin2 (anti-Axin2, Cterminus, rabbit polyclonal antibody; Abcam, Cambridge, England; 1:200), TGF- $\beta-R 1$ (V-22) (anti-TGF- $\beta$ receptor I, internal region, rabbit polyclonal antibody; Santa Cruz Biotechnology, Santa Cruz, CA; 1:50), Smad3 (Ab-425) (anti-Smad3, aa 423427, rabbit polyclonal antibody, Signalway Antibody, Pearland,TX; 1:50), p-Smad3 (Phospho-Ser425) (antiPhosphopeptide-Smad3, phosphorylation site of Ser 425, rabbit polyclonal antibody, Signalway Antibody, Pearland, TX; 1:50). Samples were then treated with a secondary antibody, peroxidaselabeled rabbit-anti-goat -IgG (H+L) (ZYMED, San Francisco, CA;
1:50), at room temperature for $1 \mathrm{~h}$ or biotin-labeled rabbit-antiIgG (Elite ABC kit: VECTASTAIN) and avidin-biotinylated peroxidase complex (ABC) reagent (Elite ABC kit: VECTASTAIN) each at room temperature for $30 \mathrm{~min}$. In addition, color was developed with a $\mathrm{Ni}^{2+}-\mathrm{Co}^{2+}-\mathrm{DAB}$ solution ${ }^{30)}$. For observations related to antibody reactions, negative control sections were produced by treating the sections with rabbit-IgG (1:50) or goat-IgG (1:50).

\section{Results}

\section{Rat first molar germ on embryonic day 19}

The germ was in the early bell stage before differentiating to odontoblast and ameloblast, showing dense mesenchymal cell distribution in the dental papilla along the high columnar inner enamel epithelium (Fig. 1a). Immunostaining with anti-Wnt10 and anti-Dvl showed positive reactions in the inner epithelium and the cervical loop. In the dental papilla, positive reactions of anti-Dvl and anti-Wnt10 were observed in mesenchymal cells in contact with the enamel organ (Fig. 1b, c). Immunostaining with anti-Axin2, anti-TGF $\beta$-R1, anti-pSmad3, and anti-Smad3 also revealed positive reactions in the inner enamel epithelium, in the cervical loop, and in the dental papilla cells in contact with the enamel epithelium, with particularly strong positive reactions shown in the inner enamel epithelium and in the cervical loop (Fig. 1e-h). Staining with anti-pSmad3 and anti-Smad3 revealed stronger positive reactions in the nucleus of inner enamel epithelium and dental papilla cells (Fig. 1g, h). In the control group, no specific reactions were observed (Fig. 1d, i).

\section{Rat maxillary first molar germ 10 days after birth}

The calcified enamel and dentin had formed in the cusp and an elongated Hertwig's epithelial sheath was present. Near the cusp, tall columnar maturation-stage ameloblasts and odontoblasts were distributed, and near the apical end in the dental neck, differentiation from preodontoblasts into odontoblasts and secretory ameloblasts was observed (Fig. 2a). Immunostaining 
Mitsuko Moriguchi et al.: Axin2 and p-Smad3 in Molar Germ

with anti-Wnt10, anti-Dvl, anti-Axin2, anti-TGFß-R1, antipSmad3, and anti-Smad3 showed uniformly strong positive reactions in odontoblasts and secretory-stage ameloblasts, moderately positive reactions in preodontoblasts in the tooth neck, and revealed stronger reactions with differentiation from preodontoblasts into odontoblasts (Fig. 2b, c, d, f, g, h). However, these immunostainings showed markedly decreased reactions in maturation-stage ameloblasts with the progress of change from the secretory stage (Fig. 2b, c, d, f, g, h). In the control group, no specific reactions were observed (Fig. 2e, i).

On the basis of these results, Wnt10, Dvl, Axin2, TGF $\beta$-R1, pSmad3, and Smad3 showed similar distribution patterns in the inner enamel epithelium, ameloblasts (secretory and maturation stages), dental papilla cells (contact with the enamel organ), preodontoblasts, and odontoblasts. Concerning ameloblasts, their distribution increased with differentiation from inner enamel epithelium into secretory-stage ameloblasts but decreased rapidly at the maturation stage. Regarding odontoblasts, their distribution increased with differentiation from dental papilla cells in contact with the enamel epithelium to odontoblasts. Axin2 and p-Smad3 in particular showed identical distribution patterns (Table 1).

\section{Discussion}

\section{Odontoblasts and the Wnt and TGF-ß signaling pathways}

In the Wnt signaling pathway in odontoblasts, Wnt-10a is reportedly involved in differentiation and tooth morphogenesis by inducing Dspp ${ }^{10)}$. In our study, all antibodies against Wnt-10, Dvl, and Axin2, which are involved in the Wnt signaling pathway, reacted with dental papilla cells adjacent to enamel epithelium, preodontoblasts, and odontoblasts, and strong reaction was noted in odontoblasts, which are at an advanced stage of differentiation. These findings indicated that Wnt-10 signals are transmitted through Dvl and Axin2 and involved in odontoblast differentiation and dentin formation by Dspp. Unclear distribution of Axin was expected because it is a negative regulator of the Wnt signaling pathway $^{2)}$ and degraded under Wnt signals ${ }^{3)}$, but it showed the strongest positive reactions in odontoblasts. Wnt signaling induces Axin2 mRNA transcription ${ }^{4}$, but induced Axin2 forms a negative feedback loop and controls Wnt signaling ${ }^{4), 5)}$, in which the Axin2 concentration is considered to show time-dependent oscillation ${ }^{311}$. However, the anti-Axin2 reaction homogeneously showed the strongest positivity over the whole region of odontoblasts, showing no fluctuation of the concentration. These findings suggested that, although Axin2 detected in odontoblasts was induced by Wnt signaling, it may have another role besides the control of Wnt through the feedback loop as a negative regulator.

Regarding Dspp in odontoblasts, there have been many reports on TGF- $\beta$, in addition to reports on Wnt-10a. TGF- $\beta-1$ has been reported to regulate Dspp through TGF- $\beta$-R1 and $\operatorname{Smad}^{23), 24)}$, and TGF- $\beta$-2 has been reported to induce Dspp ${ }^{32}$. Accordingly, it is possible that Wnt-10a-induced Dspp expression is involved in not only the Wnt but also TGF-ß signaling pathway. In our findings regarding the TGF-ß signaling pathway, Smad3 and its active type, p-Smad3, showed almost identical positive reactions in dental papilla cells adjacent to enamel epithelium, preodontoblasts, and odontoblasts, and Smad3 distributed in these cells was mostly pSmad3, suggesting that the TGF- $\beta$ signaling pathway was activated. In addition, immunoreactions of TGF-ß-R1, Smad3, and p-Smad3 in the above cells showed a similar tendency to those of Wnt10, Dvl, and Axin2, suggesting that both Wnt and TGF-ß signaling pathways are involved in these cells. .It has been reported that Axin2 interacts with Smad3 and facilitates the activation of Smad3, showing the presence of cross-talk between the Wnt and TGF- $\beta$ signaling pathways ${ }^{27}$. In our study, both Axin2 and p-Smad3 showed clear positive reactions in dental papilla cells adjacent to enamel epithelium, preodontoblasts, and odontoblasts, showing a consistent tendency of reactions accompanying the differentiation. This finding suggested that Axin2 and Smad3 are present and function in cooperation in the above cells, and cross-talk mediated by these factors may be present between the Wnt and TGF- $\beta$ signaling pathways.

\section{Ameloblasts and the Wnt and TGF- $\beta$ signaling pathways}

It has been reported that Wnt-10b distributed in mouse inner enamel epithelium and the cervical loop ${ }^{8)}$, Wnt-10a expression in incisor tooth germ preameloblasts and ameloblasts ${ }^{11)}$, and Dvl distributed in inner enamel epithelium, cervical loop, preameloblasts and ameloblasts ${ }^{12}$, so that the Dvl-mediated Wnt10 signaling pathway being involved in the proliferation of inner enamel epithelium cells and the ameloblast differentiation. In our study, anti-Wnt10 antibody showed a reaction identical to that of Dvl in the inner enamel epithelium, cervical loop, and secretorystage ameloblasts, confirming that Wnt signal transmission is mediated by Dvl in these cells. However, in maturation-stage ameloblasts, both antibodies showed weak reactions, and no involvement of the Dvl-mediated Wnt10 signaling pathway in the differentiation from the secretory to maturation stage was noted. It has been reported that a low level of Axin2 appeared in tooth germ preameloblasts and ameloblasts ${ }^{11)}$. In our study, the reaction was strongly or most strongly positive in the inner enamel epithelium, cervical loop, and secretory ameloblasts in the molar tooth germ, excluding maturation-stage ameloblasts, and no concentration fluctuation-like reaction was observed. Therefore, Axin2 may also play another role besides that in the negative feedback loop in these cells, as in odontoblasts.

Reportedly, TGF- $\beta$ is present in the inner enamel epithelium and cervical loop, is involved in tooth morphogenesis, regulates secretory ameloblast differentiation ${ }^{14)}$, and promotes amelogenin and enamelysin secretion ${ }^{20), 21)}$. In our study, TGF- $\beta$-R1, Smad3, and p-Smad3 showed strong or strongest positive reactions in the 
J.Hard Tissue Biology Vol. 21(2):113-120, 2012

inner enamel epithelium, cervical loop, and secretory ameloblasts, and the reactions of Smad3 and p-Smad3 were particularly strong in the cell nuclei in the inner enamel epithelium. Since activetype p-Smad3 forms a complex with Smad4, moves into the nucleus, and is involved in gene transcription, these findings were considered to indicate activation of the TGF- $\beta$ signaling pathway. In secretory ameloblasts, TGF-ß-R1, Smad3, and p-Smad3 showed strong or strongest positive reactions, but the reactions declined in maturation-stage ameloblasts. These findings were similar to the reaction tendencies of Wnt10, Dvl, and Axin2, and p-Smad3 and Axin2 showed identical reactions. Therefore, in the above cells excluding mature ameloblasts, it was suggested that Axin2 and Smad3 may mediate the relationship between the Wnt and TGF-ß signaling pathways, and Axin2 may be involved in the TGF-ß pathway-induced tooth morphogenesis and secretory ameloblast differentiation.

In summary, we hypothesize that, in developing rat molar, Wnt10, Dvl and Axin2 in the Wnt signaling pathway, as well as TGF$\beta$-R1 and Smad3 in the TGF- $\beta$ signaling pathway, are involved in odontoblast and ameloblast differentiation and matrix protein secretion at the secretory stage. At this time, a cross-talk between the 2 signaling pathways through Axin2 and Smad3 may be involved in that.

\section{References}

1. Logan CY and Nusse R. The Wnt signaling pathway in development and disease. Annu Rev Cell Dev Biol 20: 781810, 2004

2. Zeng L, Fagotto F, Zhang T, Hsu W, Vasicek TJ, Perry WL 3rd, Lee JJ, Tilghman SM, Gumbiner BM and Costantini F. The mouse Fused locus encodes axin, an inhibitor of the Wnt signaling pathway that regulates embryonic axis formation. Cell 90: 181-192, 1997

3. Tolwinski NS, Wehrli M, Rives A, Erdeniz N, DiNardo S and Wieschaus E. Wg/Wnt signal can be transmitted through arrow/LRP5,6 and Axin independently of Zw3/Gsk3beta activity. Dev Cell 4: 407-418, 2003

4. Jho EH, Zhang T, Domon C, Joo CK, Freund JN and Costantini F. Wnt/beta-catenin/Tcf signaling induces the transcription of Axin2, a negative regulator of the signaling pathway. Mol Cell Biol 22: 1172-1183, 2002

5. Lustig B, Jerchow B, Sachs M, Weiler S, Pietsch T, Karsten U, van de Wetering M, Clevers H, Schlag PM, Birchmeier W and Behrens J. Negative feedback loop of Wnt signaling through upregulation of conductin/axin2 in colorectal and liver tumors. Mol Cell Biol 22: 1184-1193, 2002

6. Sarkar L, Sharpe PT. Sarkar L and Sharpe PT. Expression of Wnt signalling pathway genes during tooth development. Mech Dev 85: 197-200, 1999

7. Kratochwil K, Galceran J, Tontsch S, Roth W and Grosschedl
R. FGF4, a direct target of LEF1 and Wnt signaling, can rescue the arrest of tooth organogenesis in Lef1(-/-) mice. Genes Dev 16: 3173-3185, 2002

8. Nadiri A, Kuchler-Bopp S, Haikel Y and Lesot H. Immunolocalization of BMP-2/-4, FGF-4, and WNT10b in the Developing Mouse First Lower Molar. J Histochem Cytochem 52: 103-112, 2004

9. Liu F, Chu EY, Watt B, Zhang Y, Gallant NM, Andl T, Yang SH, Lu MM, Piccolo S, Schmidt-Ullrich R, Taketo MM, Morrisey EE, Atit R, Dlugosz AA and Millar SE. Wnt/betacatenin signaling directs multiple stages of tooth morphogenesis. Dev Biol 313: 210-224, 2008

10. Yamashiro T, Zheng L, Shitaku Y, Saito M, Tsubakimoto T, Takada K, Takano-Yamamoto T and Thesleff I. Wnt10a regulates dentin sialo- phosphoprotein mRNA expression and possibly links odontoblast differentiation and tooth morphogenesis. Differentiation 75: 452-462, 2007

11. Suomalainen M and Thesleff I. Patterns of Wnt pathway activity in the mouse incisor indicate absence of Wnt/betacatenin signaling in the epithelial stem cells. Dev Dyn 239: 364-372, 2010

12. Moriguchi M, Yamada M, Miake $\mathrm{Y}$ and Nitta $\mathrm{Y}$. Immunolocalization of the factors related to Wnt signaling pathway in developing rat molar. J Hard Tissue Biol 20: 185-194, 2011

13. Tian H. Lv P, Ma K, Zhou C and Gao X. Beta-catenin/LEF1 activated enamelin expression in ameloblast-like cells. Biochem Biophys Res Commun 398: 519-524, 2010

14. Vaahtokari A, Vainio S and Thesleff I. Associations between transforming growth factor beta 1 RNA expression and epithelial-mesenchymal interactions during tooth morphogenesis. Development 113: 985-994, 1991

15. Jepsen S, Schiltz P, Strong DD, Scharla SH, Snead ML and Finkelman RD. Transforming growth factor-beta 1 mRNA in neonatal ovine molars visualized by in situ hybridization: potential role for the stratum intermedium. Arch Oral Biol 37: 645-653, 1992

16. Smith AJ, Matthews JB and Hall RC. Transforming growth factor-ß-1 (TGF- $\beta-1$ ) in dentin matrix. Ligand activation and receptor expression. Eur J Oral Sci 106: 179-184, 1998

17. Moriguchi M, Yamada M, Miake Y and Yanagisawa T. Immunolocalization of TAK1, TAB1, and p38 in the developing rat molar. Anat Sci Int 86: 69-77, 2011

18. Unda FJ, Martin A, Hilario E, Be'gue-Kirn C, Ruch JV and Are'chaga J. Dissection of the odontoblast differentiation process in vitro by a combination of FGF1, FGF2 and TGFß-1. Dev Dyn 218: 480-489, 2000

19. Coin R, Haikel Y and Ruch JV. Effects of apatite, transforming growth factor beta-1, bone morphogenetic protein-2 and interleukin-7 on ameloblast differentiation in 
Mitsuko Moriguchi et al.: Axin2 and p-Smad3 in Molar Germ

vitro. Eur J Oral Sci 107: 487-495, 1999

20. Nakata A, Kameda T, Nagai H, Ikegami K, Duan Y, Terada $\mathrm{K}$ and Sugiyama T. Establishment and characterization of a spontaneously immortalized mouse ameloblast-lineage cell line. Biochem Biophys Res Commun 308: 834-839, 2003

21. Gao Y, Li D, Han T, Sun Y and Zhang J. TGF- $\beta 1$ and TGF$\beta$-R1 are expressed in ameloblasts and promote MMP20 expression. Anat Rec (Hoboken) 292: 885-90, 2009

22. Melin M, Joffre-Romeas A, Farges JC, Couble ML, Magoloire $\mathrm{H}$ and Bleicher F. Effects of TGF beta1 on dental pulp cells in cultured human tooth slices. J Dent Res 79: 1689-1696, 2000

23. Unterbrink A, O'Sullivan M, Chen S and MacDougall M. TGF beta-1 down regulates DMP-1 and DSPP in odontoblasts. Connect Tissue Res 43: 354-358, 2002

24. He WX, Niu ZY, Zhao SL, Jin WL, Gao J and Smith AJ. TGF-beta activated Smad signalling leads to a Smad3mediated down-regulation of DSPP in an odontoblast cell line. Arch Oral Biol 49: 911-918, 2004

25. Xu X, Jeong L, Han J, Ito Y, Bringas PJ and Chai Y. Developmental expression of Smad1-7 suggests critical function of TGF- $\beta$ /BMP signaling in regulating epithelialmesenchymal interaction during tooth morphogenesis. Int $\mathrm{J}$ Dev Biol 47: 31-39, 2003
26. Yokozeki M, Afanador E, Nishi M, Kaneko K, Shimokawa H, Yokote K, Deng C, Tsuchida K, Sugino H and Moriyama $\mathrm{K}$. Smad3 is required for enamel biomineralization. Biochem Biophys Res Commun 305: 684-690, 2003

27. Furuhashi M, Yagi K, Yamamoto H, Furukawa Y, Shimada S, Nakamura Y, Kikuchi A, Miyazono K and Kato M. Axin facilitates Smad3 activation in the transforming growth factor beta signaling pathway. Mol Cell Biol 21: 5132-5141, 2001

28. Guo X, Ramirez A, Waddell DS, Li Z, Liu X and Wang XF. Axin and GSK3-ß control Smad3 protein stability and modulate TGF-ß signaling. Genes Dev 22: 106-120, 2008

29. Scott JE and Kyffin TW. Demineralization in organic solvents by alkylammonium salts of ethylenediamine- tetraacetic acid. Biochem J 169: 697-701, 1978

30. Adams JC. Heavy metal intensification of DAB-based HRP reaction product. J Histochem Cytochem 29: 775, 1981

31. Jensen PB, Pedersen L, Krishna S and Jensen MH. A Wnt oscillator model for somitogenesis. Biophys J 98: 943-950, 2010

32. Oka S, Oka K, Xu X, Sasaki T, Bringas P Jr and Chai Y. Cell autonomous requirement for TGF-beta signaling during odontoblast differentiation and dentin matrix formation. Mech Dev 124: 409-415, 2007 
J.Hard Tissue Biology Vol. 21(2):113-120, 2012 